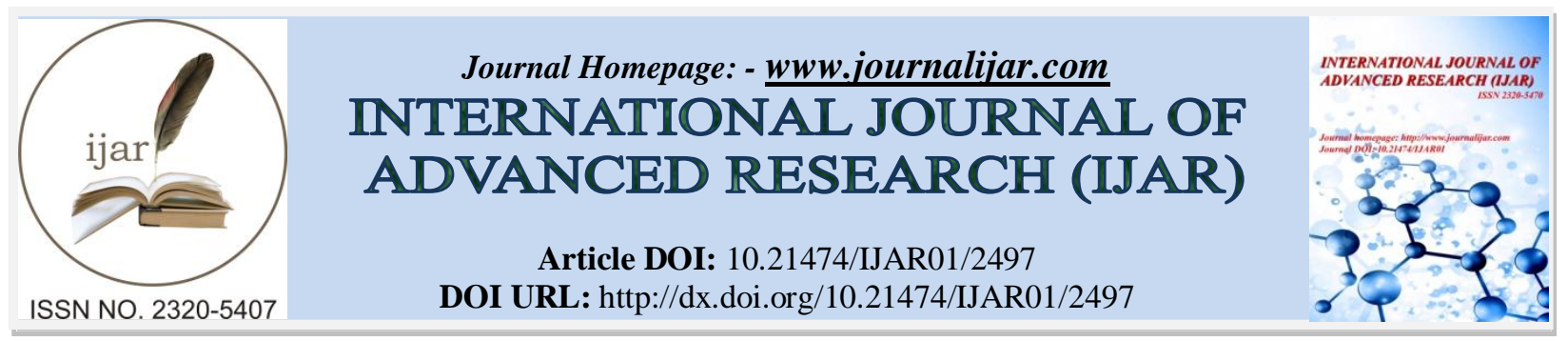

RESEARCH ARTICLE

\title{
AN ATTEMPT TO REDUCE THE TOXICITY OF BISPHENOL A BY USING PROBIOTIC DRUG THERAPY - A STUDY OF 2 D PAGE.
}

\author{
S.preethi ' and *Dr. J.Pramoda kumari ${ }^{2}$
}

Department of Microbiology, S.V. University, Tirupati, Andhra Pradesh, India

\section{Manuscript Info}

Manuscript History

Received: 25 October 2016

Final Accepted: 23 November 2016

Published: December 2016

Key words:-

Bisphenol A, Protein profiles, 2-D

electrophoresis, Scatter plots and

correlations.

\section{Abstract}

Bisphenol A (BPA) is a chemical extensively used in the manufacture of polycarbonate plastics and epoxy-phenolic resins. The human population is widely exposed to BPA, primarily by way of the diet by migration from food and beverage containers. In the present study, we were isolated the hypothalamus and liver tissues of healthy Rattus norvergicus, treated with BPA and BPA with probiotic bacteria Lactobacillus salivarius. The proteins were extracted from the tissues. The isolated proteins were washed with 2-D clean up kit because it is very sensitive to salts and detergents and the protein concentration was quantified for loading the samples in 2-D electrophoresis. Proteins were separated based on IEF and Molecular weight. The gels were Silver stained to observe the protein spots and were scanned by using Epson Expression 11000XL Scanner. We noticed over all 1237 unique proteins in our gels, in that 62 protein spots were isolated. Over all 26 protein spots showed over expression and remaining 36 protein spots showed under expression. Most of the over expressed proteins were identified in Bisphenol treated liver tissue and most of the under expressed proteins were noticed in Bisphenol with probiotic treated hypothalamus. Scattered plots were drawn between the obtained protein match IDs. Further, this study helps to the functional analysis through MALDI-TOF.

Copy Right, IJAR, 2016,. All rights reserved.

\section{Introduction:-}

BPA is one of the most highly produced industrial chemicals globally. It is used primarily for the manufacture of polycarbonate plastic and epoxy resins and used in a wide variety of consumer products [1]. BPA may be present in small quantities as unreactive monomer in some materials, and may leach from some food contact substances and polycarbonate products as the material ages or is exposed to harsh chemicals such as dish washing detergents or is exposed to UV radiation [2]. A number of effects of BPA in animals have been broadly investigated and target organs identified in repeat-dose animal studies include intestine, liver and kidney [3]. 2-D gel electrophoresis is derived from 1-D SDS-PAGE, and expands the number of proteins resolved on an electrophoresis gel by separating the proteins based on their native charge and molecular mass [4, 5]. This technique has been widely used and successfully applied in a variety of biological systems. The proteins are finally visualized by radio labeling or detected with a variety of staining methods such as silver, coomassie blue or fluorescent stains. Adapted image capture devices are used to generate digital images that can be analyzed with 2-D gel software such as Image Master 2-D platinum [6]. Every spot on the ensuing two dimensional arrays corresponds to one protein species within the 
sample. Thousands of various proteomes will therefore be separated, and knowledge like the proteome, the apparent mass, and also the quantity of every protein is obtained [7]. By the technique of $2 \mathrm{DE}$ both the over expressed and under expression is analyzed. Under expression is the process by which a cell decreases the quantity of a cellular component, such as RNA or protein, in response to an external variable that may be either a chemical or dust and increase of a cellular protein is called up-regulation. A scatter plot is a graph that has a horizontal axis ( $x$-axis) and a vertical axis ( $y$-axis) with one variable is plotted on each axis. These scatter plots are drawn between the obtained protein spot IDs and Line graphs are like scatter plots in that they record individual data values as marks on the graph.

\section{Materials and Methods:- Sample collection:-}

Adult male ( $\mathrm{n}=48$ ) (body weight $190 \pm 10 \mathrm{~g}$ ) Sprague Dawley rats were purchased from SRIVENKATESWARA TRADERS, BANGALORE, INDIA. The rats were fed on Pellet diet (Godrej agrovet ltd,Mumbai, India) and water ad libitum, maintained under standard laboratory conditions (temperature, $22-25^{\circ} \mathrm{C}$; light: dark cycle, 12:12 hr). Rats were administered orally with low and high doses of bisphenol A and the weights of animals were decreased.The effects of BPA were observed on various tissue parameters of brain and liver organs. The male Sprague dawley rats were sacrificed on $4{ }^{\text {th }}$ week of experiment [8]. The hippocampal regions of the brain were dissected and then stored at $-80^{\circ} \mathrm{C}$ until use. The abdominal cavities of the anesthesitized rats were immediately opened and the livers were excised, perfuse with normal saline to remove blood, blotted between filter papers and used for the preparation of tissue homogenate [9].

Protein Extraction from hypothalamus and liver Tissue:-

$1 \mathrm{gm}$ of both the hypothalamus and liver tissue were weighed and grinded in 50mM Tris Buffer. Homogenizations of the samples were carried out and the samples were centrifuged at 14,000 rpm. The supernatants obtained in both the individual tissues were collected into separate tubes. To the pellet, urea lyses buffer was added. The dissolved sample was now mixed with the obtained supernatant. TCA precipitation was carried out.

\section{D Procedure Employed:-}

The same samples were used for loading onto 2DE. 50 $\mu \mathrm{L}$ of sample was used for Liver control, Bisphenol treated Liver and Bisphenol+Probiotic treated Liver. $100 \mu \mathrm{L}$ of the sample was used for Hypothalamus control, Bisphenol treated Hypothalamus and Bisphenol+Probiotic treated Hypothalmus. The samples were loaded on to the IEF strips 3-10pH Linear, $18 \mathrm{~cm}$ and kept for Iso-Electric Focusing. After IEF run, the strip was equilibrated in Equilibration Buffer and the second dimension was carried out on a 10\% SDS-PAGE. The gels were Silver stained to observe the protein spots and were scanned using Epson Expression 11000XL Scanner. The image analysis of the gels was carried out using Image Master 2D Platinum 7.

\section{Image scanning and analysis:-}

All destained gels were digitized using gel scanner (Typhon Variable Mode imager), and allowed for gel analysis by using Image master 2-D platinum 6.0 software programme. It quantifies the protein spots, and showed the variation between the control and treated gel samples, the spot size indicates, up-regulation and down-regulation of the protein. The expressed protein spots were separated by using spot cutter and these spots can be analyzed by MS- for protein identification. 


\section{Results and Discussion:-}

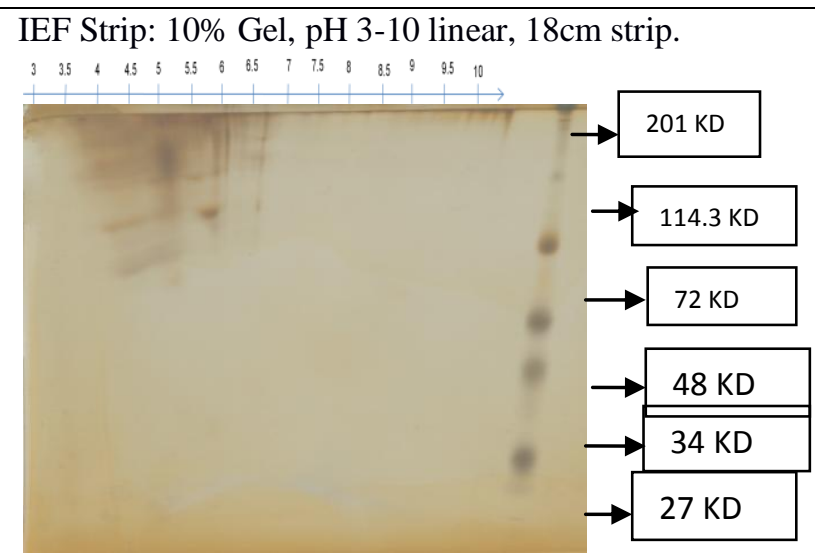

Fig 1:- 2 DE Gel Image of Hypothalamus Control tissue sample

\section{IEF Strip: 10\% Gel, pH 3-10 linear, 18cm strip.}

$\begin{array}{lllllllllllllll}3 & 3.5 & 4 & 4.5 & 5 & 5.5 & 6 & 6.5 & 7 & 7.5 & 8 & 8.5 & 9 & 9.5 & 10\end{array}$

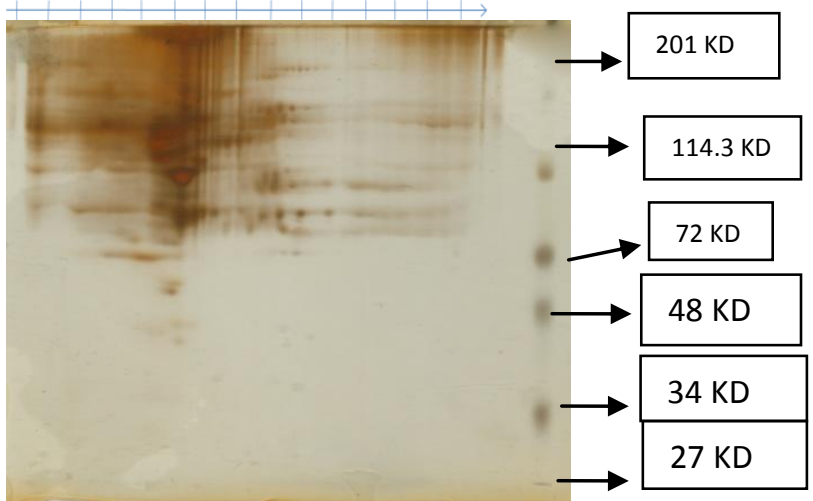

Fig2:- 2 DE Gel Image of Bisphenol induced Hypothalamus tissue sample

IEF Strip: $10 \%$ Gel, $\mathrm{pH}$ 3-10 linear, $18 \mathrm{~cm}$ strip.

$\begin{array}{lllllllllllllll}3 & 3.5 & 4 & 4.5 & 5 & 5.5 & 6 & 6.5 & 7 & 7.5 & 8 & 8.5 & 9 & 9.5 & 10\end{array}$

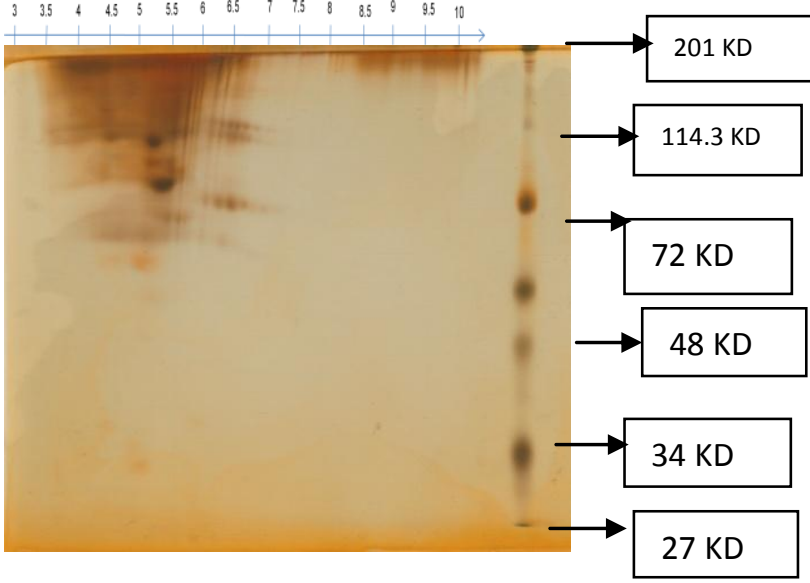

Fig 3:- 2 DE Gel Image of Bisphenol and probiotic treated Hypothalamus Control tissue sample

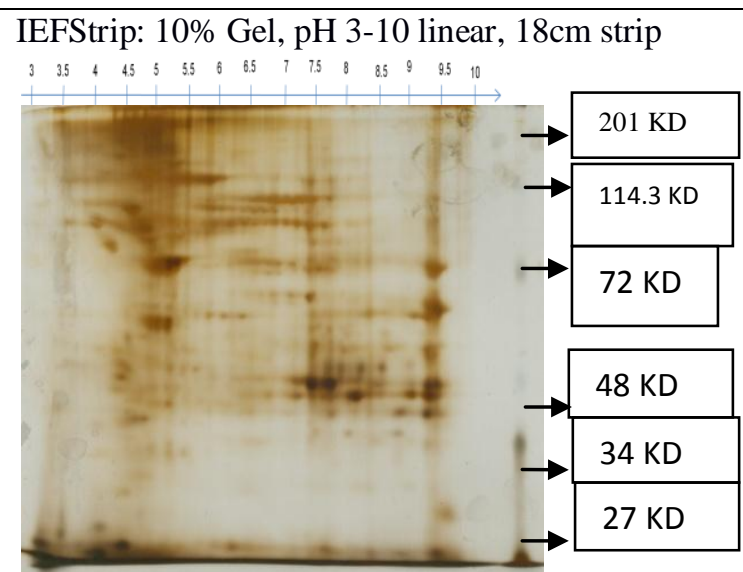

Fig 4:- 2 DE Gel Image of Liver Control tissue sample

IEF Strip: 10\% Gel, pH 3-10 linear, 18cm strip

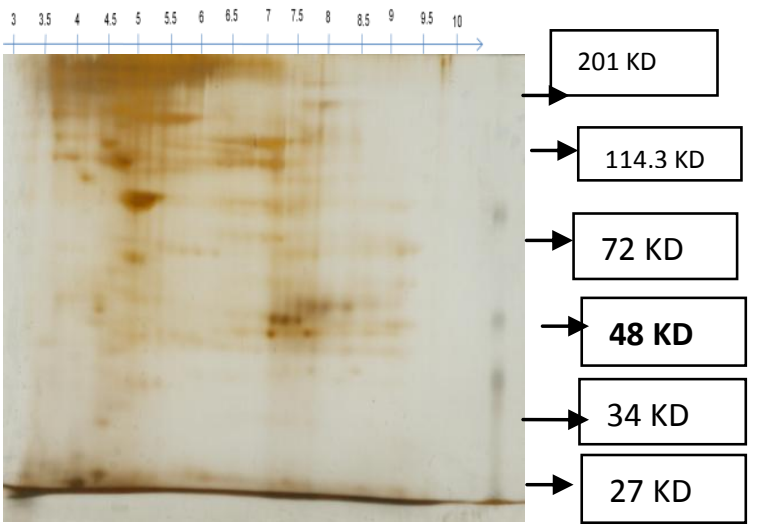

Fig 5:- 2 DE Gel Image of Bisphenol induced liver tissue sample.

\section{IEF Strip: 10\% Gel, $\mathrm{pH}$ 3-10 linear, 18cm strip}

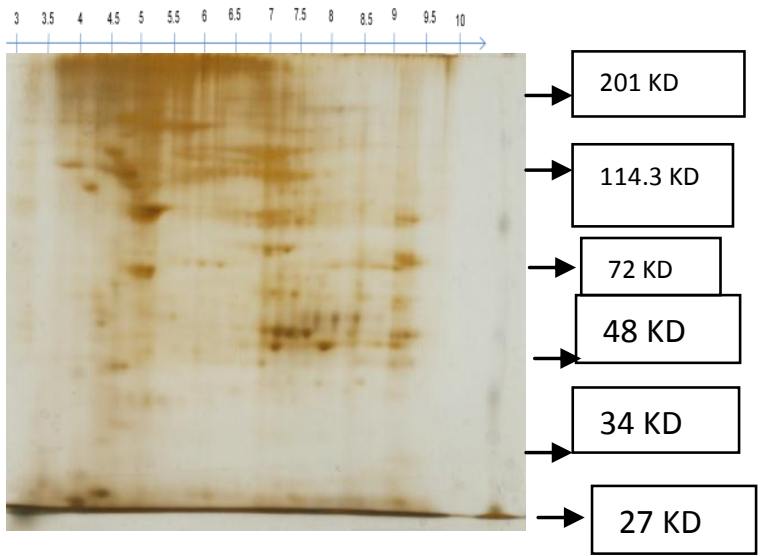

Fig 6:- 2 DE Gel Image of Bisphenol and probiotic treated liver tissue sample. 


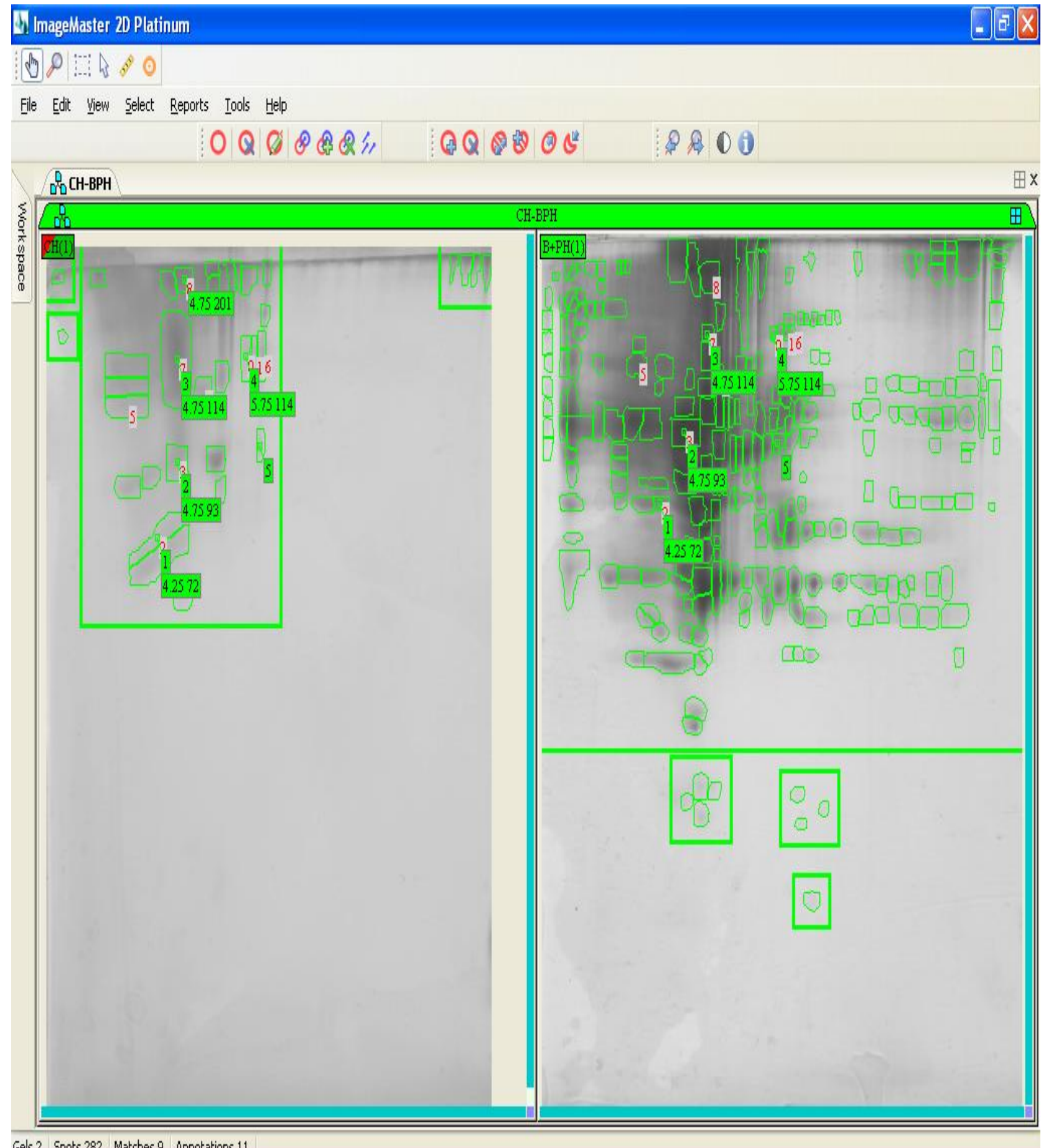

Gels 2 Spots 282 Matches 9 Annotations 11

Fig 7:- Two dimensional gel protein spot analysis by using image master 2-d platinum 6.0 software programme Match IDs of proteins in control hypothalamus with comparison to bisphenol with probiotic induced hypothalamus sample are marked on the gel 


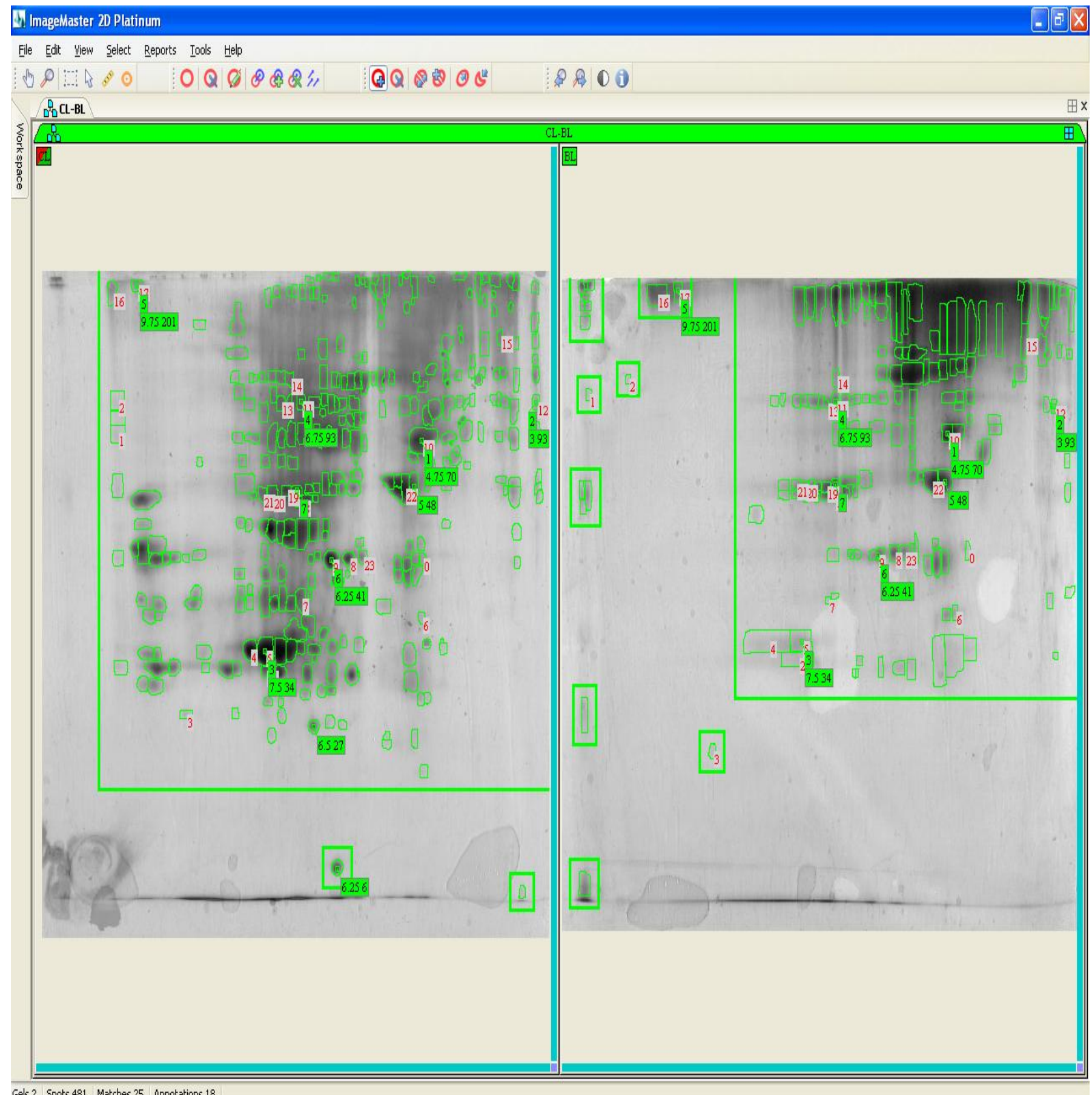

Spots 481 Matches 25 Annotations 18

Fig 8:- Two dimensional gel protein spot analysis by using image master 2-d platinum 6.0 software programme. Match IDs of proteins in liver control with comparison to bisphenol induced liver sample are marked on the gel 


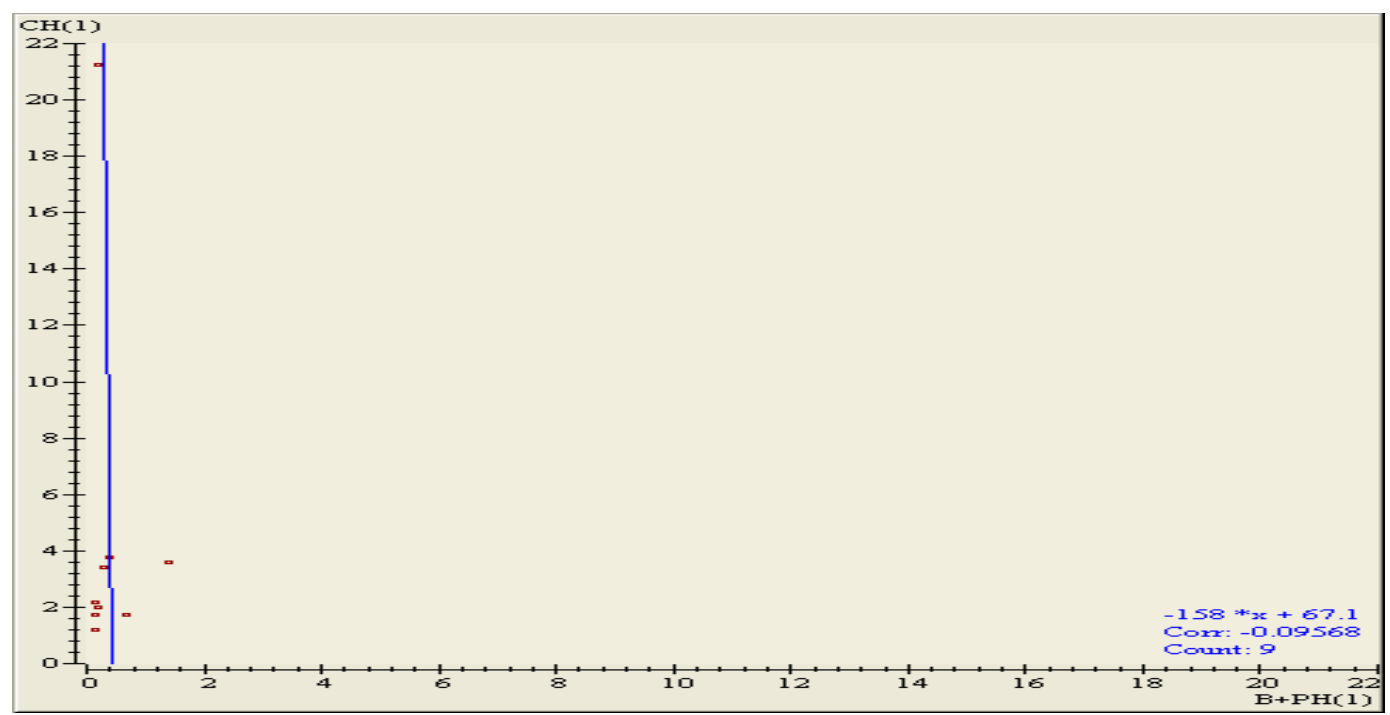

Fig 9:- Scatter plot of protein match IDs of Hypothalamus control sample and bisphenol with probiotic sample.Scatter plot for two images Control Hypothalamus and Bisphenol and Probiotic treated Hypothalamus within the apparent pI range of 4-5 and the apparent molecular weight range of $72-201$ with Correlation value = 0.09568 (Non-linear with little weak or no correlation).

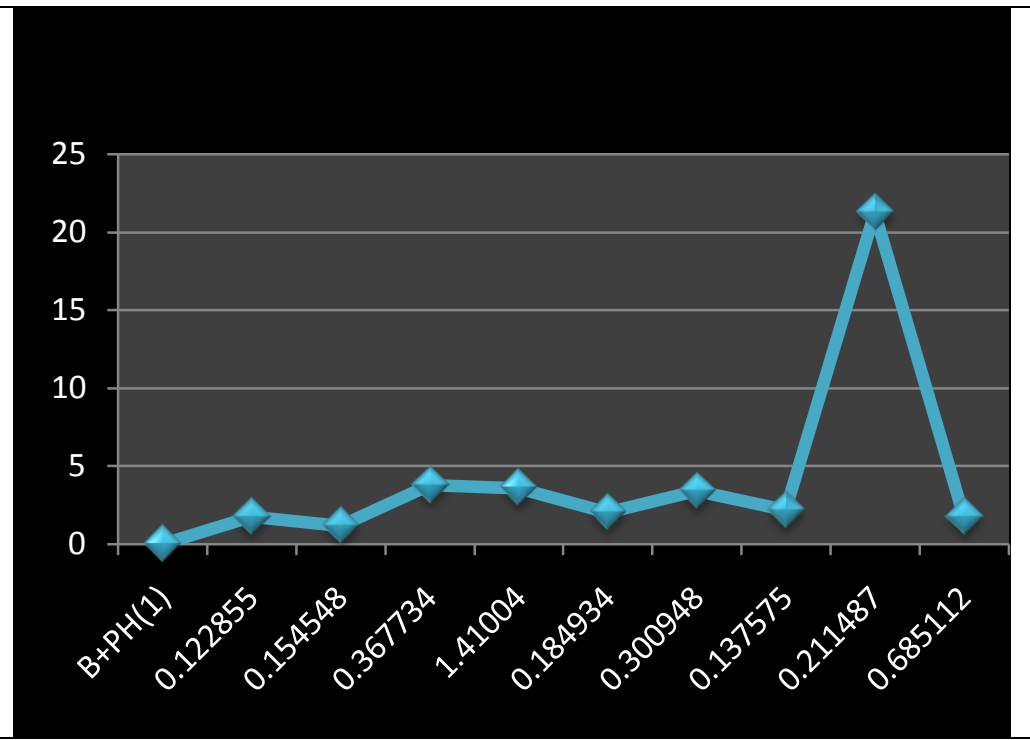

Fig 10:- A line graph representation with variables hypothalamus control on $\mathrm{Y}$-axis and bisphenol with probiotic treated hypothalamus on X-axis. 


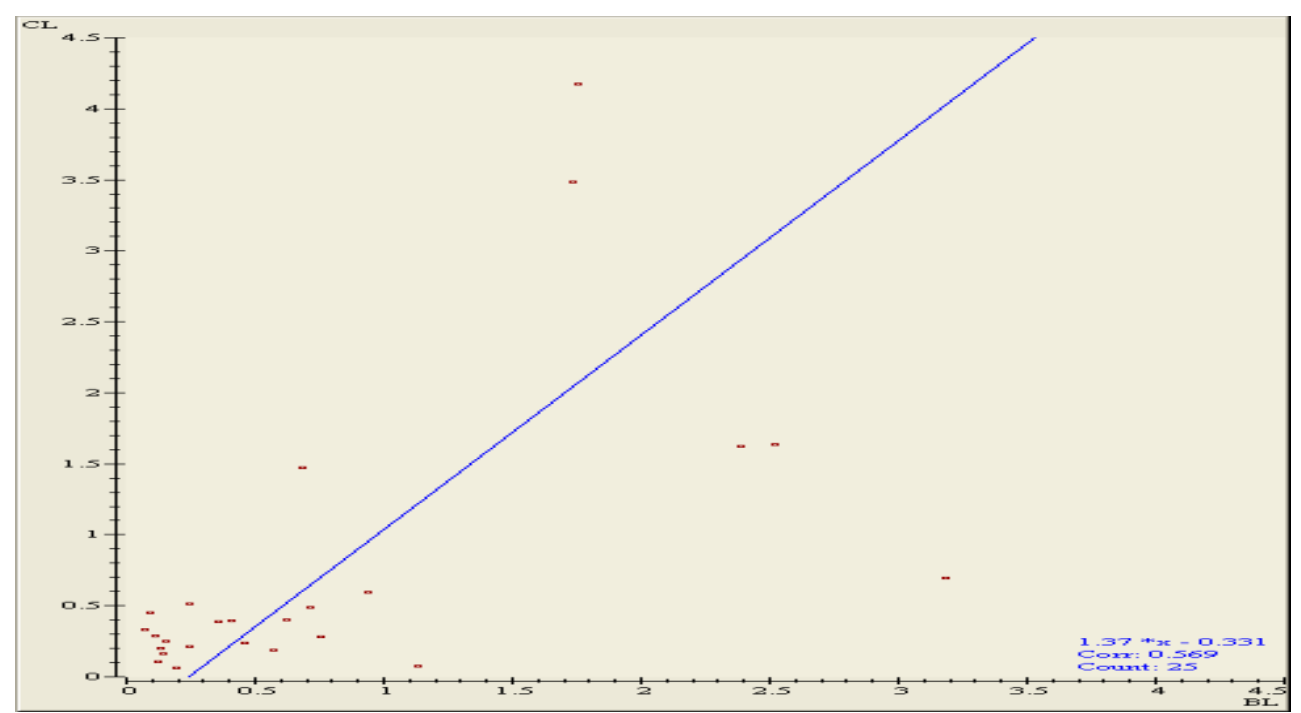

Fig 11:- Scatter plot of protein match IDs of liver control and Bisphenol treated liver sample.

Scatter plot for two images Control Liver and Bisphenol treated Liver within the apparent pI range of 3-9 and the apparent molecular weight range of 6-201 with Correlation value $=0.569$ (Non linear Positive correlation)

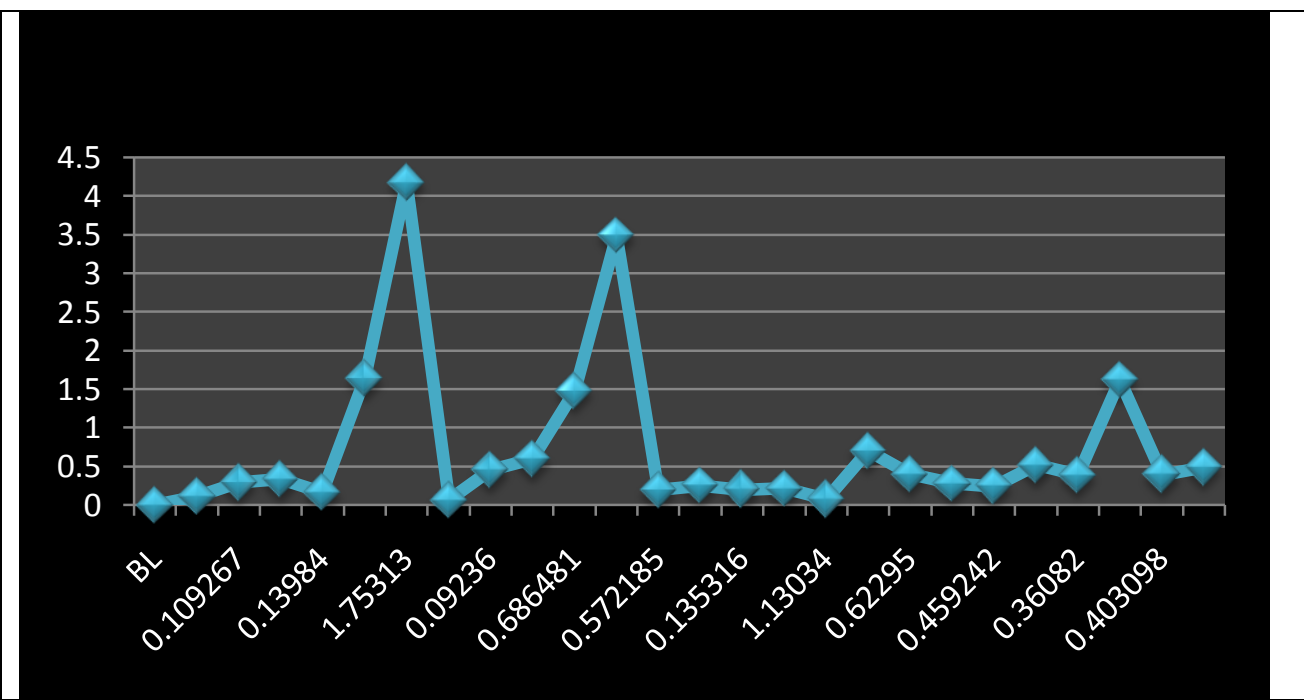

Fig 12:- A line graph representation with variables control Liver control on Y-axis and Bisphenol treated liver on $\mathrm{X}$ - axis.

\section{Discussion:-}

Figures 1 to 6 representing the 2DE gel images of hypothalamus and liver tissues of control, BPA influenced and BPA with probiotic treated Rattus norvegicus. Maximum spots of proteins shown (average value of 3.938120654) in bisphenol treated liver samples. Where, Bisphenol with probiotic gel samples showed least under expression (average value of 0.14866245) in Hypothalamus and moderate expression was observed in remaining test samples of Rattus norvegicus. Scatter plot of BPA and probiotic treated Hypothalamus tissue sample indicating non-linear weak correlation (Fig.9) and line graph showing point of correlation between two proteins (Fig.10). Scatter plot of BPA stressed liver tissue sample indicating non-linear positive correlation (Fig.11) and line graph showing point of correlation between two proteins (Fig.12).Line graphs provide an excellent way to map independent and dependent variables that are both quantitative. When both variables are quantitative, the line segment that connects two points on the graph expresses a slope, which can be interpreted visually relative to the slope of other lines or expressed as a precise mathematical formula. Scatter plots are similar to line graphs in that they start with mapping quantitative data points 


\section{Conclusion:-}

The present study was targeted to study the protein expression on impact of Bisphenol and Bisphenol with probiotic namely Lactobcillus salivarius treated hypothalamus and liver gel samples of Rattus norvegicus. Liver is highly influenced by Bisphenol when compared to Hypothalamus. Proteome analysis revealed that nine proteins are of upregulated in the Bisphenol treated liver samples compared to control condition. Bisphenol and probiotic induced Hypothalamus tissue proteins exhibited down regulation. Up- and down-regulation of proteins might play a role in cellular homeostasis. Further, the spots are undergoing for MALDI-TOF-MS analysis and the functional analysis of proteins are to be noticed in detail.

\section{Acknowledgement:-}

The authors are thankful to UGC, New Delhi for providing financial assistance (UGC-MRP).

\section{References:-}

1. Beronius,H., Hanberg,A,2011, Sources of exposure to bisphenol A. IMM report. Institute of Environmental Medicine, Karolinska Institute: ki.se/IMM)2.

2. Calafat,A., 2011, Background Paper on BPA Biomonitoring and Biomarker Studies. World Health Organization, Geneva.

3. International Food Safety Authorities Network (INFOSAN) 5/ No 2009 - Bisphenol A.

4. Gorg, Obermaier,C., Boguth, G., Harder, A.,Scheibe, B.,Wildgrube,R., and Weiss,W.,2000 The current state of two-dimensional electrophoresis with immobilized pH gradients, Electrophoresis.21,1037-1053.

5. Hanash,S., 2000, Biomedical applications of two-dimensional electrophoresis using immobilized pH gradients: current status. Electrophoresis. 21,1202-1209.

6. Chandrasekhar,K., Sreevani,S., and Pramoda Kumari,J., 2014. Identification of E.coli nissle 1917 proteins by using 2-d gel electrophoresis under the influence of cocos nucifera sap and wine,IJPSR.5(7),2763-2771.

7. Haleem,J., et al., "Two-Dimensional polyacrylamide gel electrophoresis (2D-PAGE): advances and perspectives", BioTechniques.44(2008) 697-700

8. Pramoda Kumari,J., 2014,Toxicity Studies of Bisphenol-A Induced Sprague Dawley Rats , Ijppr.Human. 2(1) ,11-19.

9. Wessam mohammad Abdel-wahab.(2014) Thymoquine Attenuates Toxicity And Oxidative Stress Induced By Bisphenol A In Liver Of Male, Pak.biol. sci.7(11), 1152-1160. 